\title{
BMJ Open Buteyko method for people with asthma: a protocol for a systematic review and meta-analysis
}

\author{
Karla Morganna Pereira Pinto de Mendonça, ${ }^{1}$ Sean Collins, ${ }^{2}$ Tácito ZM Santos, ${ }^{1}$ \\ Gabriela Chaves, ${ }^{3}$ Sarah Leite, ${ }^{1}$ Thayla Amorim Santino (D) , 1 \\ Karolinne Souza Monteiro (i) ${ }^{4}$
}

To cite: Mendonça KMPP, Collins S, Santos TZM, et al. Buteyko method for people with asthma: a protocol for a systematic review and meta-analysis. BMJ Open 2021;11:e049213. doi:10.1136/ bmjopen-2021-049213

- Prepublication history and additional supplemental material for this paper are available online. To view these files, please visit the journal online (http://dx.doi.org/10.1136/ bmjopen-2021-049213).

Received 19 January 2021 Accepted 06 August 2021

Check for updates

(c) Author(s) (or their employer(s)) 2021. Re-use permitted under CC BY-NC. No commercial re-use. See rights and permissions. Published by BMJ.

${ }^{1}$ Department of Physical Therapy, Graduate Program in Physical Therapy, Federal University of Rio Grande do Norte, Natal, Rio Grande do Norte, Brazil

${ }^{2}$ Department of Physical Therapy, Plymouth State University, Plymouth, New Hampshire, USA

${ }^{3}$ Research and Development, Myant Inc, Toronto, Ontario, Canada

${ }^{4}$ Faculty of Health Sciences of Trairi, Federal University of Rio Grande do Norte, Santa Cruz, Rio Grande do Norte, Brazil

Correspondence to Dr Karla Morganna Pereira Pinto de Mendonça;

karla-morganna@hotmail.com

\section{ABSTRACT}

Introduction Buteyko method is recommended as a non-pharmacological treatment for people with asthma. Although the worldwide interest in the Buteyko method, there is a paucity of studies gathering evidence to support its use. Therefore, we aim to conduct a systematic review and meta-analysis to assess the effects of the Buteyko method in children and adults with asthma.

Methods and analysis We will search on Cochrane Central Register of Controlled Trials, MEDLINE, Embase, US National Institutes of Health Ongoing Trials Register ClinicalTrials.gov and WHO International Clinical Trials Registry Platform for studies focusing on the Buteyko method for children and adults with asthma. The searches will be carried out in September 2021 from database's inception to the present. We will include randomised controlled trials comparing Buteyko method alone with asthma education or inactive control intervention. There will be no restriction on language. Primary outcomes include quality of life, asthma symptoms and adverse events/side effects. Two review authors will independently screen the studies for inclusion and extract data. We will assess the quality of the included studies using the 'Risk of Bias' tool. The certainty of the evidence will be assessed using the GRADE approach. Data synthesis will be conducted using Review Manager software. Reporting of the review will follow the Preferred Reporting Items for Systematic Reviews and Meta-Analyses guidance and the Cochrane Handbook for Systematic Reviews of Interventions.

Ethics and dissemination This study will assess and provide evidence for the use of the Buteyko method in people with asthma. We will analyse secondary data and this does not require ethics approval. The findings will be published in peer-reviewed journals, at relevant conferences and will be shared in plain language in social media. Moreover, the findings of this review could guide the direction of healthcare practice and research. PROSPERO registration number CRD42020193132.

\section{INTRODUCTION}

Asthma is a chronic airway disease that is associated with airway inflammation and hyper-responsiveness. Individuals with asthma generally experience respiratory symptoms (e.g., wheeze, dyspnoea, shortness of

\section{Strengths and limitations of this study}

- This systematic review protocol follows the Preferred Reporting Items for Systematic Reviews and Meta-Analyses Protocols guidelines and the Cochrane Handbook for Systematic Interviews of Interventions.

- This study will address the gap in the current evidence by providing an assessment of the effects of the Buteyko method in children and adults with asthma.

- There will be no language restriction.

- This protocol may be limited due to the lack of patient and public involvement.

- The findings of this study will help guide researchers and clinicians to treat children and adults with asthma.

breath, chest tightness, cough) that vary in frequency and intensity, as well as variable expiratory airflow limitation that may become persistent. ${ }^{12}$ Asthma has been considered an important health problem with a complex aetiology which impacts over 300 million people worldwide. ${ }^{2}$ Besides the increased prevalence, this disease is still underdiagnosed and under treated and plays a substantial burden not only to the patients, but also to their families. ${ }^{3}$

Although there is no cure for asthma, its control can be achieved under an appropriate management. Pharmacological treatment is needed to control the progression, reduce symptoms and deaths. Besides that, nonpharmacological self-management strategies, such as breathing retraining, have been advocated as an additional strategy in evidencebased asthma guidelines. ${ }^{3} 4$ Breathing exercises and/or respiratory retraining techniques have aroused the interest of people with asthma and have been recommended in guidelines as adjuvant treatments for people whose asthma symptoms are poorly controlled despite pharmacological treatment. ${ }^{25}$ 
The Buteyko method consists of a wide set of concrete and structured techniques to retrain the involuntary mechanisms controlling breathing patterns. ${ }^{6}$ Patients are encouraged to incorporate this method of breath control in their daily life by combining breathing training and breath-holding techniques. ${ }^{7}$ The Buteyko method attempts to optimise breathing to normalise breathing volume and rate to more correctly match metabolic demands to avoid overbreathing (chronic hyperventilation) ${ }^{8}$ There are several techniques employed in practice and in the research literature including nasal breathing, reducing the breathing volume (depth), frequency (breaths per minute), velocity (speed of the air flow both in and out), alterations to timing (prolong the exhalation in relation to the inhalation), rhythm (promote even breathing flow without interruption, inhale and hold), diaphragm breathing and pauses such as including natural breaks with relaxation after exhalation. ${ }^{8}$ The patients are informed regarding the importance of nose breathing, nose-cleaning exercises, reversal of chronic hyperventilation and lifestyle changes. ${ }^{78}$ The controlled reduction in breathing is combined with periods of breath-holding, known as control pauses, and these pauses are used on self-monitoring as an objective outcome measure. ${ }^{6} 9$ The control pause gets longer in people who regularly perform the Buteyko Method and is considered a sign of increase in the dyspnoea tolerance. ${ }^{7}$

Previous systematic reviews have presented summarised evidence for breathing exercises in general, which included Buteyko method, yoga, Alexander techniques, Papworth method and evaluated separately overall outcomes for children and adults with asthma. ${ }^{10-13}$ In the perspective of the Buteyko Method, studies have reported improvements in lung function, ${ }^{14}$ asthma control ${ }^{15}$ and asthma symptoms. ${ }^{16}$ It has also been recommended as a non pharmacological approach for improving asthma symptoms in adults ${ }^{9} 1617$ and children. ${ }^{1418} 19$ However, to our knowledge, no previous systematic review has combined evidence towards the Buteyko method alone. This systematic review will carefully examine and evaluate the evidence for different asthma outcomes. We aim to point out the existing evidence of the effects of the Buteyko method in people with asthma. Thus, this will comprise an evidence base and will assist guidelines and recommendations for the treatment of these patients based on standard methods. Therefore, this systematic review aims to assess the effects of the Buteyko method in children and adults with asthma.

\section{METHODS AND ANALYSIS \\ Registration}

This protocol is reported according to the Preferred Reporting Items for Systematic Reviews and MetaAnalyses (PRISMA) Protocols statement ${ }^{20}$ and the Cochrane Handbook for Systematic Reviews of Interventions. ${ }^{21}$

\section{Eligibility criteria}

\section{Types of studies}

We will include randomised controlled trials reported in full text and studies published as an abstract only and unpublished data.

\section{Types of participants}

We will include children (18 years or younger) and adults (older than 18 years) with a diagnosis of asthma. We will exclude participants with other respiratory comorbidities, such as chronic obstructive pulmonary disease; bronchiectasis or genetic diseases, such as cystic fibrosis.

\section{Types of interventions}

We will include studies comparing Buteyko method alone with asthma education or inactive control intervention (e.g., no treatment, standard care or a waiting list control).

1. Buteyko method versus asthma education.

2. Buteyko method versus inactive control.

We will not consider the inclusion of studies in which the Buteyko Method is combined with any other intervention besides the usual pharmacological treatment.

\section{Types of outcome measures}

\section{Primary outcomes}

1. Quality of life (measured by any validated respiratory disease-specific scale).

2. Asthma symptoms (measured by any validated respiratory disease specific scale, e.g., Asthma Control Test, Childhood Asthma Control Test).

3. Adverse events/side effects.

\section{Secondary outcomes}

1. Physiological measures as partial pressure of end tidal CO2 $\left(\mathrm{PETCO}_{2}\right)$, lung function as forced expiratory volume in the first second (FEV1).

2. Airway inflammation measures (exhaled nitric oxide, induced sputum eosinophil count, IgE).

3. Hyperventilation symptoms (e.g., measured by Nijmegen Questionnaire).

4. Mental health measures (measured by any validated specific or generic scale, e.g., Hospital Anxiety and Depression Score).

Reporting one or more of the outcomes listed here in the study is not an inclusion criterion for the review.

We will report outcomes using the following time point categories:

1. Immediate.

2. Short-term (up to 3 months).

3. Long term (more than 3 months).

\section{Information sources}

Search strategy

We will conduct the searches in September 2021. We will identify studies from searches on the following databases and trial registers: 
1. Cochrane Central Register of Controlled Trials (CENTRAL), via the Cochrane Register of Studies, all years to date.

2. MEDLINE Ovid SP 1946 to date.

3. Embase Ovid SP 1974 to date.

4. US National Institutes of Health Ongoing Trials Register ClinicalTrials.gov (www.clinicaltrials.gov).

5. WHO International Clinical Trials Registry Platform ( apps.who.int/trialsearch).

The proposed MEDLINE search strategy is listed in online supplemental file 1 . This will be adapted for use in the other databases.

All databases and trials registries will be searched from their inception to the present, and there will be no restriction on language or type of publication. Handsearched conference abstracts and grey literature will be identified through the CENTRAL database.

\section{Searching other resources}

We will check the reference lists of all primary studies and review articles for additional references. We will search for errata or retractions from included studies published in fulltext on PubMed and report the date this was done within the review.

\section{Data collection and analysis \\ Selection of studies}

The search results will be imported into the reference list management tool Mendeley (https://www.mendeley. com). Any duplicates will be identified and removed using Mendeley. Then, the reference list will be exported to the Rayyan QCRI systematic review web-based application (https://rayyan.qcri.org). ${ }^{22}$ We will record the selection process in sufficient detail to complete a PRISMA flow diagram. ${ }^{23}$

Two review authors (TZMS and SL) will screen the titles and abstracts of the remaining search results independently and code them as 'retrieve' (eligible or potentially eligible/unclear) or 'do not retrieve'. We will retrieve the full-text study reports of all potentially eligible studies and two review authors (TZMS and SL) will independently screen them for inclusion, recording the reasons for exclusion of ineligible studies. We will resolve any disagreement through discussion or, if required, we will consult a third review author (SC). We will identify and exclude duplicates and collate multiple reports of the same study so that each study, rather than each report, is the unit of interest in the review.

\section{Data extraction and management}

We will extract data for all included studies using a prepiloted form. Two review authors (KSM and TAS) will extract the following study characteristics from included studies:

1. Methods: study design, total duration of study, details of any 'run-in' period, number of study centres and location, study setting, withdrawals and date of study.
2. Participants: $\mathrm{N}$, mean age, age range, gender, severity of condition, diagnostic criteria, baseline lung function, smoking history, inclusion criteria and exclusion criteria.

3. Interventions: intervention, comparison, duration of intervention, method of delivery.

4. Outcomes: primary and secondary outcomes specified and collected, and time points reported.

5. Notes: funding for studies and notable conflicts of interest of trial authors.

Two review authors (KSM and TAS) will independently extract outcome data from included studies. We will note in the 'Characteristics of included studies' table if outcome data were not reported in a usable way. We will resolve disagreements by consensus or by involving a third review author (KMPPdM). One review author (TAS) will transfer data into the Review Manager (RevMan 2020). We will double-check that data are entered correctly by comparing the data presented in the systematic review with the study reports. A second review author (GC) will spot-check study characteristics for accuracy against the study report.

\section{Assessment of risk of bias in included studies}

The risk of bias for each study will be independently assessed by two review authors (TZMS and SL). We will use version two of the Cochrane 'Risk of Bias' tool (RoB 2), outlined in the Cochrane Handbook for Systematic Reviews of Interventions. ${ }^{24}$ Any disagreement will be resolved by a third author (GC). We will assess the risk of bias according to the following domains:

1. Bias arising from the randomisation process.

2. Bias due to deviations from intended interventions.

3. Bias due to missing outcome data.

4. Bias in measurement of the outcome.

5. Bias in selection of the reported result.

We will judge each potential source of bias as high, low or some concerns and provide a quote from the study report together with a justification for our judgement in the 'Risk of bias' table. The judgements of each domain listed will be also summarised in the 'Risk of bias' table. The overall risk of bias for the result is the least favourable assessment across the domains of bias. We will consider blinding separately for different key outcomes where necessary. Information from unpublished data or correspondence with a trialist will be noted in the 'Risk of bias' table. When considering treatment effects, we will take into account the risk of bias for the studies that contribute to that outcome.

\section{Assessment of bias in conducting the systematic review}

We will conduct the review according to this published protocol and justify any deviations from it in the 'Differences between protocol and review' section of the systematic review.

\section{Measures of treatment effect}

Dichotomous data will be analysed as ORs and continuous data as the mean difference (MD) or standardised 
MD. In case data from rating scales are combined in a meta-analysis, we will confirm they are inputted with a consistent direction of effect (e.g., higher scores indicating improvement).

We will pool the data in meta-analyses when relevant; that means, if the treatments, participants, and the underlying clinical question are similar enough so the metaanalyses will make sense.

We will describe skewed data as medians and IQRs for each group.

Where multiple trial arms are reported in a single study, we will include only the relevant arms. If two comparisons (e.g., drug A vs placebo and drug B vs placebo) are combined in the same meta-analysis, we will either combine the active arms or halve the control group to avoid double-counting. ${ }^{25}$

Adjusted analyses (analysis of variance [ANOVA] or analysis of covariance [ANCOVA]), if available, will be used in our meta-analyses. If both change from baseline and endpoint scores are available for continuous data, we will use change from baseline unless there is low correlation between measurements in individuals. If a study reports outcomes at multiple time points, we will consider the short term, medium term and long term. ${ }^{25}$

Intention-to-treat or 'full analysis set' analyses will be used, if reported, instead of completer or per-protocol analyses.

\section{Unit of analysis issues}

For dichotomous outcomes, we will use participants, rather than events, as the unit of analysis (i.e., number of participants having adverse events, rather than number of adverse events per participant). However, if rate ratios are reported in a study, we will analyse them on this basis.

\section{Dealing with missing data}

We will contact investigators or study sponsors in order to verify key study characteristics and obtain missing numerical outcome data where possible (e.g., when a study is identified as an abstract only or in case of unavailable data). Where this is not possible, and the missing data are thought to introduce serious bias, we will consider this in the Grading of Recommendations Assessment, Development and Evaluation (GRADE) rating for affected outcomes.

\section{Assessment of heterogeneity}

We will use the $\mathrm{I}^{2}$ statistic to measure heterogeneity among the studies in each analysis according to the guidance in the Cochrane Handbook for Systematic Reviews of Interventions. $^{21}$ If we identify substantial heterogeneity, we will report it and explore the possible causes by prespecified subgroup analysis.

\section{Assessment of reporting biases}

If we are able to pool more than 10 studies, we will create and examine a funnel plot to explore possible small study and publication biases.

\section{Data synthesis}

We will use a random-effects model and perform a sensitivity analysis with a fixed-effect model.

\section{Subgroup analysis and investigation of heterogeneity}

We plan to carry out the following subgroup analyses:

1. Degree of asthma severity: mild versus moderate-tosevere, as defined by the Global Initiative for Asthma. ${ }^{2}$

2. Duration of treatment (immediate to short term vs long term).

We will use the following outcomes in subgroup analyses:

1. Quality of life.

2. Asthma symptoms.

We will use the formal test for subgroup interactions in Review Manager V.5 (Revman). ${ }^{26}$

\section{Sensitivity analysis}

We plan to carry out a sensitivity analysis in which we only include studies with an overall low risk of bias or some concerns, excluding studies at high risk of bias. We will also compare the results from a fixed-effects model with the random-effects models.

\section{Summary of findings and assessment of the certainty of the evidence}

We will create a 'Summary of findings' table using the following outcomes at short term (up to 3 months) point: quality of life, asthma symptoms, adverse events/side effects, physiological measures (FEV1), airway inflammation measures (exhaled nitric oxide), hyperventilation symptoms, mental health measure (anxiety). We will use the five GRADE considerations (risk of bias, consistency of effect, imprecision, indirectness and publication bias) to assess the certainty of a body of evidence as it relates to the studies that contribute data for the prespecified outcomes. We will follow the recommendations described in the Cochrane Handbook for Systematic Reviews of Interventions (Section 8.5 and Chapter 12), ${ }^{27}$ using GRADEpro software (GRADEpro GDT) ${ }^{28}$ We will justify all decisions to downgrade the quality of studies using footnotes and we will make comments to aid the reader's understanding of the review where necessary.

\section{Patient and public involvement}

This protocol was not designed with the patient or public involvement. However, people with asthma will be invited to provide feedback on the systematic review results and on the plain language summaries that will be created to disseminate the findings.

\section{ETHICS AND DISSEMINATION}

This systematic review will assess and provide evidence for the use of Buteyko method in children and adults with asthma. No ethical approval is required because only publicly available and published data will be analysed.

Through the publication of the results in peer-reviewed journals and at relevant conferences, the findings of this 
review could guide the direction of healthcare practice and research. The results will also be divulged in plain language in social media to spread the knowledge with the society and the public interested in the topic.

Acknowledgements We would like to thank Federal University of Rio Grande do Norte and Coordenação de Aperfeiçoamento de Pessoal de Nível Superior (CAPES) for supporting this study.

Contributors KMPPdM, SC, TAS and KSM conceptualised and designed the protocol, drafted the initial manuscript and reviewed the manuscript. TAS developed the search strategy. GC, TZMS and SL defined data extraction process and methodological appraisal of the studies. GC planned statistical analysis. All authors approved and contributed to the final written manuscript.

Funding KMPPdM and KSM receive support in the form of salaries from Federal University of Rio Grande do Norte, Brazil. TAS is a PhD scholarship holder funded by Coordenação de Aperfeiçoamento de Pessoal de Nível Superior (CAPES), BrazilFinance Code 001.

Competing interests KMPPdM has an academic interest in breathing exercises (including Buteyko) and has been chief investigator on two trials evaluating Buteyko method in children with asthma. TAS was a coinvestigator on two trials evaluating Buteyko method in children with asthma. KSM was a coinvestigator on one trial evaluating Buteyko method in children with asthma*. ${ }^{*}$ To mitigate the potential biases in the review process, review authors who were investigators on the potentially eligible studies will not be involved in the inclusion or exclusion of studies, assessment of risk of bias and GRADE the overall quality of outcomes to which the study contributed.

Patient consent for publication Not applicable.

Provenance and peer review Not commissioned; externally peer reviewed.

Supplemental material This content has been supplied by the author(s). It has not been vetted by BMJ Publishing Group Limited (BMJ) and may not have been peer-reviewed. Any opinions or recommendations discussed are solely those of the author(s) and are not endorsed by BMJ. BMJ disclaims all liability and responsibility arising from any reliance placed on the content. Where the content includes any translated material, BMJ does not warrant the accuracy and reliability of the translations (including but not limited to local regulations, clinical guidelines, terminology, drug names and drug dosages), and is not responsible for any error and/or omissions arising from translation and adaptation or otherwise.

Open access This is an open access article distributed in accordance with the Creative Commons Attribution Non Commercial (CC BY-NC 4.0) license, which permits others to distribute, remix, adapt, build upon this work non-commercially, and license their derivative works on different terms, provided the original work is properly cited, appropriate credit is given, any changes made indicated, and the use is non-commercial. See: http://creativecommons.org/licenses/by-nc/4.0/.

ORCID iDs

Thayla Amorim Santino http://orcid.org/0000-0002-5514-762X

Karolinne Souza Monteiro http://orcid.org/0000-0003-2254-8723

\section{REFERENCES}

1 Moraes T, Sears M, Subbarao P. Epidemiology of asthma and influence of ethnicity. Semin Respir Crit Care Med 2018;39:003-11.

2 Global Initiative for Asthma (GINA). Global strategy for asthma management and prevention 2020, 2020. Available: ginasthma.org/ gina-reports/ [Accessed 30 Dec 2020].

3 World Health Organization (WHO). Asthma, 2020. Available: www. who.int/news-room/fact-sheets/detail/asthma [Accessed 5 Jan 2021].

4 Bruton A, Lee A, Yardley L, et al. Physiotherapy breathing retraining for asthma: a randomised controlled trial. Lancet Respir Med 2018;6:19-28.

5 British Thoracic Society (BTS). SIGN 158 British guideline on the management of asthma: a national clinical guideline. Available: www.brit-thoracic.org.uk/quality-improvement/guidelines/asthma/ [Accessed 5 Jan 2021].

6 Bruton A, Lewith GT. The Buteyko breathing technique for asthma: a review. Complement Ther Med 2005;13:41-6.

7 Courtney R. Buteyko breathing method. In: Chaitow L, Bradley DGC, eds. Recognizing and treating breathing disorders: a multidisciplinary approach. 2nd editio. Toronto: Elsevier Health Sciences, 2014. : 241-7p..

8 Sakharoff M. Buteyko Breathing Technique and Ketogenic Diet as Potential Hormetins in Nonpharmacological Metabolic Approaches to Health and Longevity. In: The science of hormesis in health and longevity, 2018: 257-74.

9 Mohamed Y, Elderiny S, Ibrahim L. The effect of Buteyko breathing technique among patients with bronchial asthma: comparative study 2019;2:2.

10 Connor E, Patnode CD, Burda BU, et al. Breathing exercises and/ or retraining techniques in the treatment of asthma: comparative effectiveness. Rockville (MD): Agency for Healthcare Research and Quality (US), 2012.

11 Sankar J, Das RR. Asthma - a disease of how we breathe: role of breathing exercises and Pranayam. Indian J Pediatr 2018;85:905-10.

12 Santino TA, Chaves GS, Freitas DA, et al. Breathing exercises for adults with asthma. Cochrane Database Syst Rev 2020;3:CD001277.

13 Macêdo TM FDA, Chaves GS, Holloway EA, et al. Breathing exercises for children with asthma. Cochrane Database Syst Rev 2016;12:4.

14 Elnaggar RK, Shendy MA. Efficacy of noninvasive respiratory techniques in the treatment of children with bronchial asthma: a randomized controlled trial. Bull Fac Phys Ther 2016;21:1-10.

15 Cowie RL, Conley DP, Underwood MF, et al. A randomised controlled trial of the Buteyko technique as an adjunct to conventional management of asthma. Respir Med 2008;102:726-32.

16 Hassan ZM, Riad NM, Ahmed FH. Effect of Buteyko breathing technique on patients with bronchial asthma. Egypt $J$ Chest Dis Tuberc 2012;61:235-41.

17 Prem V, Sahoo RC, Adhikari P. Comparison of the effects of Buteyko and pranayama breathing techniques on quality of life in patients with asthma - a randomized controlled trial. Clin Rehabil 2013;27:133-41.

18 Hepworth C, Sinha I, Saint GL, et al. Assessing the impact of breathing retraining on asthma symptoms and dysfunctional breathing in children. Pediatr Pulmonol 2019;54:706-12.

19 McHugh P, Duncan B, Houghton F. Buteyko breathing technique and asthma in children: a case series. N Z Med J 2006;119:1234.

20 Moher D, Shamseer L, Clarke M, et al. Preferred reporting items for systematic review and meta-analysis protocols (PRISMA-P) 2015 statement. Syst Rev 2015;4:1.

21 Higgins JPT, Thomas J, Chandler J. Cochrane Handbook for systematic reviews of interventions version 6.2, 2021. www.training. cochrane.org/handbook

22 Ouzzani M, Hammady H, Fedorowicz Z, et al. Rayyan-a web and mobile APP for systematic reviews. Syst Rev 2016;5:210.

23 Moher D, Liberati A, Tetzlaff J, et al. Preferred reporting items for systematic reviews and meta-analyses: the PRISMA statement. PLOS Med 2009;6:7.

24 Higgins JPT, Savović J, Page MJ. Chapter 8: Assessing risk of bias in a randomized trial. In: Higgins JPT, Thomas J, Chandler J, eds. Cochrane Handbook for systematic reviews of interventions version 6.2, 2021. www.training.cochrane.org/handbook

25 Higgins JPT, Li T, Deeks JJ. Chapter 6: Choosing effect measures and computing estimates of effect. In: Higgins JPT, Thomas J, Chandler J, et al, eds. Cochrane Handbook for systematic reviews of interventions version 6.2, 2021. www.training.cochrane.org/ handbook

26 The Cochrane Collaboration. Review Manager (RevMan) [Computer program]. Version 5.3. Copenhagen: The Nordic Cochrane Centre, 2014.

27 McKenzie JE, Brennan SE. Chapter 12: Synthesizing and presenting findings using other methods. In: Higgins JPT, Thomas J, Chandler J, et al, eds. Cochrane Handbook for systematic reviews of interventions version 6.2, 2021. www.training.cochrane.org/ handbook

28 GRADEpro GDT. GRADE Working group, McMaster University, version accessed prior to 13 August 2019. Hamilton, ON McMaster University (developed by Evidence Prime); 2015. https://gradepro. org/ 